\title{
Application of care bundles in bowel preparation for colonoscopy in children
}

\author{
Yuanyuan Liang ${ }^{1}$, Wenqiong Xin ${ }^{1}$, Yang Yang ${ }^{1}$, Xiaoling Li $^{2}$ \\ ${ }^{1}$ Department of Pediatric Surgery, West China Hospital, Sichuan University, Chengdu 610041, China; ${ }^{2}$ West China Hospital/West China School of \\ Nursing, Sichuan University, Chengdu 610041, China \\ Contributions: (I) Conception and design: Y Liang, X Li; (II) Administrative support: X Li; (III) Provision of study materials or patients: Y Liang, W \\ Xin, Y Yang; (IV) Collection and assembly of data: Y Liang, Y Yang; (V) Data analysis and interpretation: Y Liang, Y Yang; (VI) Manuscript writing: \\ All authors; (VII) Final approval of manuscript: All authors. \\ Correspondence to: Xiaoling Li. West China Hospital/West China School of Nursing, Sichuan University, Chengdu 610041, China. \\ Email: janifer818@163.com.
}

Background: To explore the value of care bundles (CBs) in bowel preparation for colonoscopy in children. Methods: Children who underwent electronic fiberoptic colonoscopy or enteroscopic surgery in our hospital from September 2016 to October 2017 were enrolled as the conventional nursing (CN) group and children who received such procedures from November 2017 to December 2018 were enrolled as the care bundle group. Polyethylene glycol electrolyte lavage solution (PEG-ELS) was used for bowel preparation in all children. The CBs included nurse education, risk evaluation of inadequate bowel preparation, education of children and families, and observation and assessment during preparation. The quality of bowel preparation, tolerance and safety, families' anxiety score, and degree of satisfaction with hospitalization were compared between these two groups.

Results: Eighty-two children were enrolled in this study, with 42 cases in the CB group and 40 cases in the CN group. Symptoms of distension, abdominal pain, vomiting, and fatigue, along with intragastric feeding, were compared between the two groups. An additional enema was performed in 2 cases in the CB group and in 12 cases in the CN group, demonstrating a significant difference between the groups. The Aronchick score and anxiety score of families were $1.24 \pm 0.85$ vs. $2.35 \pm 1.76(\mathrm{t}=-3.477, \mathrm{P}=0.001)$ and $3.28 \pm 0.85$ vs. $5.45 \pm 1.78(\mathrm{t}=-3.473, \mathrm{P}=0.001)$ in the $\mathrm{CN}$ group and $\mathrm{CB}$ group, respectively. The satisfaction rate was $97.62 \%$ vs. $85.00 \%\left(\chi^{2}=6.764, \mathrm{P}<0.001\right)$.

Conclusions: Implementation of the care bundles in the bowel preparation of children planning to receive colonoscopy can improve the quality of preparation and the satisfaction with hospitalization while alleviating the anxiety of patients and their families.

Keywords: Care bundles (CBs); pediatric colonoscopy; bowel preparation

Submitted May 06, 2019. Accepted for publication Aug 08, 2019.

doi: 10.21037/apm.2019.09.01

View this article at: http://dx.doi.org/10.21037/apm.2019.09.01

\section{Introduction}

There are numerous colorectal diseases in children, and their overall incidence has been on the rise. With the increased use of digestive endoscopy in clinical settings, the role of colonoscopy in the diagnosis and treatment of intestinal diseases in pediatric patients has increasingly been recognized. Bowel preparation is the most important step before enteroscopy. The quality of bowel preparation plays a key role in ensuring the accuracy of endoscopic diagnosis and the safety of treatment. Inadequate bowel preparation before enteroscopy will lead to the concealment of lesions, contamination of the endoscope surface, and influence endoscope insertion and observation; these are all the main causes of missed diagnoses and treatment failure after 
endoscopic procedures. In addition, enteroscopy in children is carried out under general anesthesia; a failed examination will not only increase the risk and pain of children but also affect the emotions of families, resulting in a waste of medical resources (1-4). At present, most of the commonly used enema solutions have a peculiar taste and are used in a large dosage, which can induce vomiting (3). Children are often unwilling to take oral enema solutions and their tolerance to the adverse reactions is poor. Therefore, bowel preparation in children is particularly difficult. Unfortunately, no uniform bowel preparation and management protocol has been available for pediatric patients. In many centers, bowel preparation for children is performed according to the requirements for adult patients. However, children are substantially different from adults physiologically, pathologically, and psychologically. The techniques and methods for adults are obviously not always suitable for children. In our current study, we tried to explore a safe and effective approach for bowel preparation so as to improve the quality of bowel preparation for children and ensure the accuracy and safety of enteroscopic diagnosis and treatment.

\section{Methods}

\section{Subjects}

Children who underwent electronic fiberoptic colonoscopy or enteroscopic surgery in our hospital from September 2016 to December 2018 were enrolled as the subjects. The inclusion criteria were as follows: (I) aged 2-14 years; (II) without any bowel preparation before admission; (III) participant families without previous history of psychotic disorder (e.g., anxiety); (IV) signed informed consent forms. The exclusion criteria were as follows: (I) presence of severe underlying diseases (such as heart, brain, and/ or lung disease, and organ dysfunction), digestive tract malformation, and intellectual development disorder; (II) caregivers incapable of effectively reading and understanding the patient education materials; (III) need for emergency enteroscopy due to foreign body in digestive tract, massive hemorrhage in digestive tract, or other reasons; (IV) lack of consent from children and/or families to participate in the study.

\section{Patients grouping and intervention}

Children who underwent electronic fiberoptic colonoscopy or enteroscopic surgery in our hospital from September
2016 to October 2017 were enrolled as the conventional nursing $(\mathrm{CN})$ group, and children who received such procedures from November 2017 to December 2018 were enrolled as the care bundle (CB) group. Polyethylene glycol electrolyte lavage solution (PEG-ELS) was used for bowel preparation in all children. The CBs included nurse education, risk evaluation of inadequate bowel preparation, children and family education, and monitoring during preparation, which were implemented after November 2017. The quality of bowel preparation, tolerance and safety, parents' anxiety score, and degree of satisfaction were compared between these two groups.

The informed consent was obtained from all patients took part in the study. All collected data was kept secure. This study was approved by the West China Hospital Research Ethics Boards.

\section{Bowel preparation protocol}

The same bowel preparation protocol was applied in both the $\mathrm{CN}$ and $\mathrm{CB}$ groups. Low-residue liquid diet was given two days before operation, followed by oral administration of PEG-ELS at a dose of $100 \mathrm{~mL} / \mathrm{kg} 12 \mathrm{~h}$ before surgery, with a maximum dose of no more than $4 \mathrm{~L}$. The solution was administered within 6-8 h. The children were fasted for $4 \mathrm{~h}$ before the examination. Additional dose, intragastric feeding, or enema was given, according to the doctor's advice, to children who could not tolerate the protocol or in whom the bowel preparation had not met the requirements.

\section{Nursing protocol in the $\mathrm{CN}$ group}

$\mathrm{CN}$ protocol was applied in the $\mathrm{CN}$ group. After the children were admitted, the responsible nurse delivered the currently used How to Prepare for Your Surgery to the patients or their families and asked questions raised by the families of the children. The families described the defecation situation on the morning of the operation.

\section{The CBs adopted in the $\mathrm{CB}$ group Training of nursing staff}

Endoscopists and experienced endoscopy nurses were invited to train all the nurses in the ward. The training focused on the following aspects: application and implementation of pediatric enteroscopy; purpose, requirements, and value of preoperative bowel preparation for pediatric enteroscopy; selection and use of commonly used drugs for pre-operative bowel preparation and their complications; and observation and evaluation of the effectiveness of bowel preparation. The training course lasted $20-30 \mathrm{~min}$ and was repeated 
three times so as to ensure all the ward nurses participated in the training. The training efficiency was evaluated by an examination with an attempt to ensure that all the trainees had understood and mastered the training content. Nurses who failed to pass the examination were asked to take a second training course or not to be involved in the implementation of the $\mathrm{CN}$ protocol.

Assessment of the risk of poor bowel preparation

After the children were admitted, the trained nurses assessed the risk of poor bowel preparation in children planning to undergo bowel preparation based on factors including Bristol stool scale type (1-7), defecation frequency, dietary intake two days before admission, medication status, previous history of enteroscopy, and previous history of bowel preparation before an examination. Children at high risk of poor bowel preparation were recorded and reported to the physician in charge, and a decision of adjusting the preparation protocol was made accordingly.

\section{Education of children and families}

For children aged 7 years or older, education was offered to both the children and their parents. For children younger than 7 years, education was offered to the parents only. The education methods included (I) on-site instruction and (II) education videos. The on-site instruction was conducted by a trained nurse using both video and images to ensure that each child and his/her families read and understood the content of the instruction. The education video offered a checklist of preparations for children's enteroscopy to the children and their families. It listed the timing and contents of the preparations from admission to discharge. There was a QR code on the picture which allowed the children and their families to watch enteroscopy-related education videos. The contents of the education videos included the value of enteroscopy, the importance of adequate bowel preparation before enteroscopy, diet during preparation, education methods, observation of defecation, possible discomforts during preparation and their management, and fasting requirements before surgery.

\section{Observation and evaluation during bowel preparation}

Bedside observation and evaluation by nurses during bowel preparation were listed as the fourth element of the CB protocol. The contents of observation and recording included (I) dietary intake in the first two days and (II) the first medication taken under the supervision of nurses, and any drug-related reactions. Unwillingness to take the drug or drug intolerance was recorded and reported to the physician in charge in a timely manner, and a decision to adjust the medication plan or change to intragastric feeding was made accordingly. After the children started defecation, the nurses came to the bedside to evaluate the defecation pattern and then recorded the defecation status in the morning in a standard form. For children with inadequate bowel preparation, the condition was reported to the endoscopist and physician in charge to decide whether to take supplementary measures or postpone enteroscopy.

\section{Supervision of the CB protocol}

After the children were admitted, all the CB measures taken by nurses for the children and their families were recorded on the nursing record sheet. The nursing record sheet included four sections: admission assessment, education and instruction, bedside disease assessment by the nurses, and defecation assessment on the morning of surgery.

\section{Evaluation indicators}

\section{Evaluation of the quality of bowel preparation}

(I) The nurses came to the bedside to evaluate the defecation on the morning of surgery. An enema was needed if the pre-operative bowel preparation did not meet the requirements. Therefore, the number of patients requiring enema before colonoscopy in these two groups was used as one of the indicators for assessing the quality of bowel preparation;

(II) An endoscopist evaluated the bowel preparation after the endoscopy was initiated by using the Aronchick scale which has the following ratings (rated from 1 to 5 points): excellent (greater than $95 \%$ of the intestinal mucosa seen), good (a large volume of clear liquid covering $25 \%$ or more of the intestinal mucosa but greater than $90 \%$ of the intestinal mucosa seen), fair (some semisolid stool that can be suctioned or washed away, but greater than $90 \%$ of the intestinal mucosa seen), poor (semisolid stool that cannot be suctioned or washed away and less than $90 \%$ of the intestinal mucosa seen), or inadequate (repeat preparation needed).

\section{Evaluation of the safety and tolerance of bowel preparation}

The symptoms of intolerance such as distension, abdominal pain, vomiting, and fatigue in addition to intragastric feeding were analyzed in both groups.

\section{Scoring of the anxiety of families}

The anxiety of families was scored using a self-designed questionnaire according to the Symptom Checklist 90 (SCL-90). The SCL-90 consists of 90 items. Ten items 
Table 1 Symptoms and intragastric feeding during bowel preparation

\begin{tabular}{lcccc}
\hline Symptoms & $\mathrm{CB}(\mathrm{n}=42)$ & $\mathrm{CN}(\mathrm{n}=40)$ & $\chi^{2}$ & $\mathrm{P}$ value \\
\hline Abdominal distension & 6 & 6 & 0.049 & 0.082 \\
Abdominal pain & 5 & 4 & 0.006 & 0.938 \\
Vomiting & 15 & 15 & 0.004 & 0.951 \\
Fatigue & 6 & 7 & 0.009 & 0.924 \\
Intragastric feeding & 6 & 6 & 0.049 & 0.825
\end{tabular}

$\mathrm{CB}$, care bundle group; $\mathrm{CN}$, conventional nursing group.

related with anxiety were selected, and the total score ranged from 0 to 40 . A higher score meant more severe anxiety, and vice versa. Two investigators carried out surveys on the families on the day of discharge. Both had received uniform training and were well informed about the purpose and significance of the survey. The selected families had cared for the children for more than $8 \mathrm{~h}$ a day and had no previous history of psychosis or anxiety. Before starting the scoring, the investigators explained the questions to the families without any hint or bias, allowing the respondents to evaluate themselves.

\section{Satisfaction with hospitalization}

On the day of discharge, the families were asked to fill in the self-made Questionnaire on the Satisfaction of Medical Services for Inpatients, which was based on the content of the patient satisfaction survey prescribed by the former Ministry of Health and the Department of Health and reflects the inputs of medical staff and management experts of our hospital. The questionnaire has a Cronbach's alpha coefficient of 0.971 and a content validity index of 0.720 . The families must be able to correctly understand the content of the questionnaire and their time spent caring for children during hospitalization must be more than $70 \%$ of the hospitalization duration. The results of hospitalization satisfaction scoring were divided into excellent $(>90 \%)$, good $(70-89 \%)$, and poor $(<70 \%)$ according to the ratio of the score of each questionnaire form over the total score. The proportion of questionnaire forms with an "excellent" or "good" result was the satisfaction rate.

\section{Statistical analysis}

Data were processed using SPSS 22.0 software. Count data are expressed as cases and percentages and are compared by chi square test or Mann-Whitney U test. The measurement data are expressed as mean \pm standard deviation, and intergroup differences were compared by using the $t$ test. A P value of $<0.05$ was considered significantly different.

\section{Results}

\section{General Information}

A total of 82 children who underwent colonoscopy or enteroscopic surgery were enrolled in this study. These patients included 60 males and 22 females, aged 2-14 years, with an average age of $4.51 \pm 3.20$ years. Among them, 42 cases were in the CB group and 40 cases were in the $\mathrm{CN}$ group. There was no significant difference between these two groups in general data such as gender, age of operation, and type of disease $(\mathrm{P}>0.05)$.

\section{Scores of bowel preparation quality in the two groups}

An additional enema was performed in 2 cases in the CB group and in 12 cases in the $\mathrm{CN}$ group, showing significant difference $(t=7.512, \mathrm{P}=0.006)$. According to the results of the Aronchick scoring, the CB group had a significantly lower score than the $\mathrm{CN}$ group $[(1.24 \pm 0.85) v s$. $(2.35 \pm 1.76)$; $\mathrm{t}=-3.477, \mathrm{P}=0.001]$. Among them, all the children in the CB group had a successful examination or operation after the first attempt. In contrast, 3 children in the $\mathrm{CN}$ group underwent repeated bowel preparation and the examination or surgery succeeded in the second attempt; a 3-year-old female child did not receive surgery before her discharge upon her family's request.

\section{Symptoms during bowel preparation}

The symptoms such as distension, abdominal pain, vomiting, and fatigue, in addition to intragastric feeding, are summarized in Table 1.

\section{Scores of the anxiety of families}

The degree of anxiety was lower in the CB group than in the $\mathrm{CN}$ group, and the anxiety score was significantly lower in the CB group than in the $\mathrm{CN}$ group $(3.28 \pm 0.85$ vs. $5.45 \pm 1.78$ respectively; $t=-3.473, \mathrm{P}=0.001$ ).

\section{Scores of hospitalization satisfaction}

The hospitalization satisfaction score in the CB group was 
Table 2 Scores of hospitalization satisfaction

\begin{tabular}{lcll}
\hline Group & Excellent (\%) & Good & Poor (\%) \\
\hline CB $(n=42)$ & $38(90.48)$ & $3(7.14)$ & $1(2.38)$ \\
CN $(n=40)$ & $25(62.50)$ & $9(22.50)$ & $6(15.00)$ \\
\hline
\end{tabular}

$\chi^{2}=6.764, \mathrm{P}<0.001$. CB, care bundle group; $\mathrm{CN}$, conventional nursing group.

significantly superior to that in the $\mathrm{CN}$ group $(97.62 \%$ vs. $85.00 \%$ respectively; $\left.\chi^{2}=6.764, \mathrm{P}<0.001\right)($ Table 2$)$.

\section{Discussion}

A CB is a collection of a series of evidence-based and interrelated interventions to form a nursing protocol for managing difficult clinical diseases (5). Such a protocol often consists of 3-5 simple, clear, and operable evidencebased measures, which can be accepted by clinical practice and have robust operability, and their joint implementation is better than any single approach in improving patient outcomes. The application of CB can dramatically increase the access of patients to the required nursing care and enable the effective implementation of each link of bowel preparation. Our current study has demonstrated the positive correlations among the elements of the $\mathrm{CB}$ protocol. Education and training of nurses is the precondition to ensure the accurate implementation of other elements. Bedside observation and evaluation by nurses is a supplement to both risk assessment and education of children's families. Timely discovery of incorrect behaviors of children and their families during bowel preparation or immediately assisting in completing the unfinished steps is very helpful. Meanwhile, observing and recording the evaluation indicators during bowel preparation also ensures the implementation of bowel preparation protocol. Therefore, although the bowel preparation protocol is the same, different supervision and implementation approaches may result in diverse clinical outcomes. The concept of $\mathrm{CB}$ has more advantages in improving the quality of bowel preparation in children.

As shown in our current study, the development of CB protocols should be strictly evidence-based:

(I) Many studies have found that the education of nurses can improve clinical outcomes. The trained nurses can reduce the uncertainty and lack of confidence in dealing with clinical problems and provide effective nursing and correct advice to patients $(6,7)$. As the first element of the $\mathrm{CB}$ protocols, the training of nurses can remarkably increase the satisfaction with hospitalization and alleviate the anxiety of parents and their families during bowel preparation. The possible reasons may include the following: (i) since the medical staff have received uniform training, the information (including cartoon education videos) concerning bowel preparation delivered by medical staff at all levels is highly consistent, which greatly improves the confidence of families; (ii) the use of nursing record sheets urges nurses to implement all aspects of the CB protocol, and their focus on the whole $\mathrm{CB}$ protocol and their active interventions reduce families' helplessness; (iii) nurses have adequate knowledge on enteroscopy and can offer detailed answers to the questions raised by families during bowel preparation, thus increasing the success rate of bowel preparation;

(II) Screening various risk factors before bowel preparation is an important basis for the education of children and their families, and also a rationale for adjusting the initial screening program. Studies suggest that the risk of poor bowel preparation is predictable (8-10). Assessment before bowel preparation allows for the timely adjustment of the bowel preparation plan in patients for whom completing bowel preparation may be difficult, whereas close observation during bowel preparation can improve the quality of the preparation. Therefore, it is essential to screen a variety of risk factors before bowel preparation and incorporate the risk assessment in the bowel preparation protocol. In our current study, nurses assessed the risk of poor bowel preparation at admission, which was highly valuable for the smooth implementation of the bowel preparation protocol;

(III) As demonstrated in many randomized controlled trials and meta-analyses, adequate oral and written education, along with communication, is an important component in the successful implementation of bowel preparation. Sun et al. (11) gave written instructions to patients' families about the appointment of hospitalization time, diets, and medication precautions, so as to improve medication compliance among children. In addition, Davis et al. (12) found that redesigning bowel preparation instructions with easier-to-read text could improve the success rate of 
bowel preparation. In our center, strengthening the education of patients and their families via multiple education modes based on both video and images was the third element of the protocol;

(IV) Bedside observation and evaluation by nurses is a supplement to both risk assessment and education of children's families. It helps in the timely discovery of any incorrect behaviors of children and their families during bowel preparation or assists in completing the unfinished steps. The patient's description of his/her last defecation during bowel preparation is unreliable (13). Therefore, bedside assessment by medical staff using standard forms is required. Johnston et al. (14) designed a simple spreadsheet to score stools from three aspects: texture, color, and sediment. Further measures were taken according to the scoring results, thus reducing the possibility of repeated enteroscopy. Families who are extremely anxious or worried about bowel preparation failure or have experience in bowel preparation should be identified. They often make too radical demands on defecation. In such situations, bedside assessment and observation by nurses is even more important. The possibility that the families perform bowel preparation by themselves in advance or increase the oral dose of oral enema solution should be considered. Education of patients and their families can be focused on the advantages of this protocol and the communication among patients, so as to enhance their confidence in the bowel preparation protocol and thus improve the accuracy of its implementation;

(V) As an invasive procedure, an enema not only brings an unpleasant experience to children but also may cause rectal mucosal injury and bleeding, which may affect the accuracy of enteroscopy. In severe cases, it may even lead to complications such as massive hemorrhage due to polyp exfoliation and intestinal perforation $(15,16)$. Observation and evaluation during multi-channel education and preparation can reduce the probability of an enema before operation. In the $\mathrm{CN}$ group, the bowel preparation protocol was poorly implemented due to the lack of observation and evaluation during bowel preparation and the lack of intensive education via multiple approaches; as a result, up to $30 \%$ of the patients underwent enema.

\section{Conclusions}

The application of CB measures in the bowel preparation for pediatric patients improves the quality of bowel preparation, which ensures the smooth, safe, and effective completion of the examination, reduces the pain and socioeconomic burden of repeated or aborted procedures due to inadequate bowel preparation, and improves the experience and satisfaction of children and their families. The CB protocol is practicable in the nursing and management of bowel preparation before enteroscopy in children and can be widely used in clinical settings.

\section{Acknowledgments}

None.

\section{Footnote}

Conflicts of Interest: The authors have no conflicts of interest to declare.

Ethical statement: The authors are accountable for all aspects of the work in ensuring that questions related to the accuracy or integrity of any part of the work are appropriately investigated and resolved. This study was approved by the West China Research Ethics Boards. Written informed consent for participation was obtained from each participant.

\section{References}

1. ASGE Standards of Practice Committee, Lightdale JR, Acosta R, et al. Modifications in endoscopic practice for pediatric patients. Gastrointest Endosc 2014;79:699-710.

2. Zmora O, Pikarsky AJ, Wexner SD. Bowel preparation for colorectal surgery. Dis Colon Rectum 2001;44:1537-49.

3. Li P, Zhang ST, Wu YD, et al. A prospective randomized comparison of oral polyethylene glycol and sodium phosphate lavage for colonoscopy preparation. J Clin Gastroenterol 2009;21:81-4.

4. Zhang JZ, Pan SC, Heng CR, et al. editors. Practical Pediatric Surgery. Hangzhou: Zhejiang Science and Technology Publishing House, 2003:38.

5. Shan J, Zhu JH, Gu YH. Advances in the concept of care bundles and its clinical application. J Nurs Train 2010;25:889-91.

6. Coleman LK, Wilson AS. Impact of Nursing Education 
on the Proportion of Appropriately Drawn Vancomycin Trough Concentrations. J Pharm Pract 2016;29:472-4.

7. Corl DE, McCliment S, Thompson RE, et al. Efficacy of diabetes nurse expert team program to improve nursing confidence and expertise in caring for hospitalized patients with diabetes mellitus. J Nurses Prof Dev 2014;30:134-42.

8. ASGE Standards of Practice Committee, Saltzman JR, Cash BD, et al. Bowel preparation before colonoscopy. Gastrointest Endosc 2015;81:781-94.

9. Hassan C, Fuccio L, Bruno M, et al. A predictive model identifies patients most likely to have inadequate bowel preparation for colonoscopy. Clin Gastroenterol Hepatol 2012;10:501-6.

10. Nguyen DL, Wieland M. Risk factors predictive of poor quality preparation during average risk colonoscopy screening: the importance of health literacy. J Gastrointestin Liver Dis 2010;19:369-72.

11. Sun XY, Xu LQ, Jiang LQ. Cause analysis and nursing countermeasures of pre-operative bowel preparation

Cite this article as: Liang Y, Xin W, Yang Y, Li X. Application of care bundles in bowel preparation for colonoscopy in children. Ann Palliat Med 2019;8(4):476-482. doi: 10.21037/ apm.2019.09.01 failure in children with colon polyps. Journal of Nurses Training 2006:740-41.

12. Davis TC, Hancock J, Morris J, et al. Impact of Health Literacy-directed Colonoscopy Bowel Preparation Instruction Sheet. Am J Health Behav 2017;41:301-8.

13. Fatima H, Johnson CS, Rex DK. Patients' description of rectal effluent and quality of bowel preparation at colonoscopy. Gastrointest Endosc 2010;71:1244-252.e2.

14. Johnston ER, Keswani RN, Cyrus R, et al. The Nursing Bowel Preparation Assessment Tool (NBPAT) Is Highly Predictive of Inpatient Bowel Preparation Adequacy: a Prospective Pilot Study. DDW 2015 ASGE Program and Abstracts 2015;81:AB154.

15. Tang YF. Prevention and nursing of complications of cleaning enema. Nurs Pract Res 2009;6:94-6.

16. Wang YE, Wu YJ, Chen YQ. Comparison the effectiveness of two cleaning enema methods. Chin Nurs Res 2012;26:148. 